\title{
RIQUEZA E DENSIDADE DE ÁRVORES, ARVORETAS E PALMEIRAS EM PARQUES URBANOS DE RECIFE, PERNAMBUCO, BRASIL
}

Leonardo Rodrigues da Silva ${ }^{1}$, Isabelle Maria Jacqueline Meunier ${ }^{2} ;$ Ângela Maria de Miranda Freitas $^{3}$

(recebido em 15.08.2007 e aceito pra publicação em 13.12.2007)

\section{RESUMO}

Neste trabalho foram reconhecidas 87 morfoespécies de árvores, arvoretas e palmeiras nos sete parques urbanos de Recife, com a observação de 2808 exemplares. As espécies de árvores e arvoretas mais abundantes foram Clitoria fairchildiana, Mangifera indica, Tecoma stans, Terminalia catappa e Tabebuia impetiginosa. Entre as palmeiras destacaram-se Dypsis lutescens, Roystonea oleracea, Cocos nucifera, Acrocomia intumescens e Elaeis guineensis. A maior abundância foi verificada no Parque Treze de Maio, com 981 exemplares e 58 espécies. O Parque da Jaqueira apresentou 773 indivíduos e 68 espécies, sendo o parque com maior riqueza de espécies. O parque menos arborizado e com menor riqueza florística foi o Robert Kennedy, com 122 indivíduos e 18 espécies, e os que apresentaram piores condições de densidade foram Arraial Novo do Bom Jesus e Santana (41,7 e 56,7 indivíduos/ha, respectivamente). O potencial paisagístico da flora local foi mal aproveitado nos parques urbanos de Recife, privilegiando-se o plantio de espécies exóticas ou nativas de outros biomas brasileiros. As densidades de árvores, arvoretas e palmeiras foram consideradas baixas em quatro dos sete parques, que precisam receber novos plantios de espécies arbóreas, preferencialmente nativas, contribuindo para amenização climática e conservação e valorização de flora nativa.

Palavras-chave: Árvores urbanas; arborização de parques.

\footnotetext{
${ }_{2}^{1}$ Agrônomo graduado pela Universidade Federal Rural de Pernambuco

2 Engenheira Florestal, M.Sc, Professora do Departamento de Ciência Florestal da UFRPE, meunier@hotlink.com.br

${ }^{3}$ Engenheira Florestal, Dra. em Botânica, Curadora do Herbário Sérgio Tavares, UFRPE
} 


\title{
RICHNESS AND ABUNDANCE OF TREES, SCRUBS AND PALMS TREES IN URBAN PARKS IN RECIFE, PERNAMBUCO, BRAZIL
}

\begin{abstract}
In this wok, we recognized 87 morphospecies of trees, shrubs and palms in seven urban parks situated in Recife, and we counted 2808 individuals. The most abundant species were Clitoria fairchildiana, Mangifera indica, Tecoma stans, Terminalia catappa and Tabebuia impetiginosa and, among palms, Dypsis lutescens, Roystonea oleracea, Cocos nucifera, Acrocomia intumescens and Elaeis guineensis. The biggest abundance of trees, shrubs and palms was found at Park 13 de Maio, with 981 individuals of 58 species. The Park da Jaqueira is the one with biggest richness of species, having 773 individuals of 68 species. The Park Robert Kennedy showed the lowest number of trees, with 122 individuals and poor richness, with 18 species, and the ones who had presented the worst conditions of density were the parks Arraial Novo do Bom Jesus and Santana (41.7 and 56.7 individuals/ha, respectively). We noticed that the paisagistic potencial of the local flora was badly used in the urban parks in Recife, where the plantation of exotic species or from others Brazilian regions was privileged. The densities of trees, shrubs and palms were considered low in four of the seven parks, in which it should be planted trees species, preferable the native ones, what will contribute to turn milder the region's climate and to native's flora conservation and valorization.
\end{abstract}

Key words: Urban trees; urban forest in parks. 


\section{INTRODUÇÃO}

Na maioria das cidades, ocupações irregulares, especulação imobiliária, construção de grandes edifícios, abertura de novas ruas e avenidas, ampliação de centros comerciais e pólos industriais e outras atividades impactantes são vistas modificando a paisagem e suprimindo áreas verdes, necessárias para se manter a qualidade do ambiente e bem estar da população.

Uma das formas de garantir as áreas verdes nos centros urbanos é estabelecer a organização dos espaços livres e neles ter assegurado pelo poder público a criação e a manutenção de parques urbanos para atender a diferentes finalidades ambientais, sociais, recreativas e contemplativas.

Por definição os parques urbanos são espaços livres públicos com função predominante de recreação, ocupando na malha urbana uma área em grau de equivalência superior à da quadra típica urbana, em geral, apresentando componentes da paisagem natural - vegetação, topografia, elemento aquático - como também edificações destinadas a atividades recreativas, culturais e/ou administrativas (CARNEIRO \& MESQUITA, 2000).

De maneira geral, os parques urbanos se mostram como unidades urbanísticas fundamentais para a vida nas cidades. Além da elevada área física, a cobertura vegetal é uma característica marcante nestes espaços, sendo um dos componentes de grande importância na formação da paisagem local. Segundo Lira (2002), a criação de parques em áreas urbanas é uma forma simples e prática de resolver problemas de poluição sonora e do ar, amenizar o clima e, entre outras funções, garantir a estabilidade emocional das pessoas.

Assim como a distribuição da energia elétrica, abastecimento de água, telefone, limpeza urbana, iluminação pública, a vegetação de uma cidade é um serviço urbano essencial (CASTRO, 2000). A vegetação promove melhoria na qualidade do ar através do processo fotossintético, pelo qual as planta absorvem $\mathrm{CO}_{2}$ e liberam $\mathrm{O}_{2}$ para atmosfera; além disso, a deposição de partículas sólidas nas folhagens das copas reduz a poluição lançada pelas fábricas, pelos veículos e pela queima de combustíveis fósseis.

Além disso, as árvores melhoram a infiltração de água no solo, pois a vegetação funciona como cobertura, reduzindo também perdas de solo por erosão hídrica. Nos centros urbanos com poucas áreas verdes, a água das chuvas que incide sobre o solo pavimentado não tem chance de infiltrar, escorrem pela superfície e ocasionam enchentes e, conseqüentemente, graves transtornos.

GATTO (2002) afirma que a vegetação serve para reduzir a velocidade dos ventos em até $80 \%$, diminuindo ou eliminando os processos erosivos em áreas descobertas, além de trazer melhorias das propriedades químicas e físicas do solo, pois os componentes das plantas são os principais formadores da matéria orgânica do solo. Essa, por sua vez, age na 
melhoria do nível de fertilidade, no aumento da capacidade de retenção de água e na manutenção da vida no solo e da própria vegetação.

Os serviços ambientais proporcionados pela cobertura vegetal em parques urbanos são, portanto, proporcionais à densidade de árvores presentes. Brandão \& Lucena (1999) mostraram, em praças do Rio de Janeiro, que em relação ao conforto térmico, a insuficiência arbórea levou a extremo desconforto térmico, inibindo as práticas de lazer ao ar livre, consideradas pelos autores como as principais funções de praças e parques.

Os parques urbanos, por contarem com áreas razoavelmente extensas e destinação voltada ao lazer contemplativo e prática de esportes, seriam espaços privilegiados para a adoção de elevado número de espécies, com ênfase àquelas da flora local, contribuindo também para a conservação e o conhecimento da biodiversidade regional. No entanto, a maioria das plantas arbóreas cultivadas em ruas, avenidas, parques e praças públicas das cidades brasileiras é formada de espécies oriundas de outros países (espécies exóticas), apesar da flora nativa contar com centenas de espécies de grande beleza e qualidade paisagística. Segundo Lorenzi (2003), as primeiras espécies exóticas introduzidas no Brasil tiveram seu emprego principalmente em parques e jardins.

Com a denominação de parques urbanos são encontrados em Recife oito espaços públicos: Parque 13 de Maio, Parque da Jaqueira, Parque Arraial Velho do Bom Jesus (Sítio da Trindade), Parque de Santana, Parque Arraial Novo do Bom Jesus (Parque do Forte), Parque Arnaldo Assunção, Parque Robert Kennedy e Parque do Caiara (SÁ CARNEIRO; MESQUITA, 2000). Os dados apresentados pelas autoras permitem estimar que os parques urbanos representam menos de $0,2 \%$ da extensão do município.

Diante da importância para a qualidade de vida das pessoas e pela sua função na conservação da biodiversidade no meio urbano, esse trabalho teve como objetivo realizar o levantamento florístico, incluindo árvores, arvoretas e palmeiras presentes em sete parques urbanos da cidade, exceto o Parque do Caiara, pela situação de abandono atualmente verificada. Além de estabelecer uma coleção botânica própria dos parques no Herbário Sérgio Tavares da UFRPE, pretendeu-se, a partir das avaliações, identificar os parques que melhor atendem aos seus objetivos socioambientais, no que se refere a densidade de árvores e riqueza de espécies, e aqueles que devem ser objeto de atenção especial, considerados como oportunidades de ampliação e melhoria do conjunto arbóreo da cidade.

\section{MATERIAL E MÉTODOS}

\section{Área de Estudo}

O estudo foi realizado entre junho de 2005 e fevereiro de 2006, na cidade do Recife, capital de Pernambuco, Brasil. Recife encontra-se numa região de clima o tipo As', de 
acordo com a classificação de Köppen, denominado tropical quente-úmido. A temperatura média oscila entre 24 e 27 graus; mesmo nos meses mais frios, julho e agosto, a temperatura média fica acima de 24 graus. O período chuvoso inicia-se em março e vai até o final de agosto (GUIA DO RECIFE, 2007).

O município do Recife possui uma área de $220 \mathrm{~km}^{2}$ e compreende atualmente 94 bairros distribuídos em seis Regiões Político-Administrativas, com uma população residente de 1.422.905 habitantes (RECIFE, 2006)

Os parques estão localizados nos bairros de Santo Amaro (Parque 13 de Maio), Jaqueira (Parque da Jaqueira), Casa Amarela (Parque Arraial Velho do Bom Jesus), Santana (Parque de Santana), Cordeiro (Parque Arraial Novo do Bom Jesus), Engenho do Meio (Parque Arnaldo Assunção) e IPSEP (Parque Robert Kennedy).

\section{Métodos da pesquisa}

Para o reconhecimento das espécies, todo o material fértil foi coletado, identificado, processado e tombado no Herbário Sérgio Tavares do Departamento de Ciências Florestais da Universidade Federal Rural de Pernambuco (HST/DCFL/UFRPE). Paralelamente ao reconhecimento, foram consultados sites e literaturas especializadas, e as espécies foram divididas em dois grupos: árvores e arvoretas, caracterizadas pela presença de caule principal lenhoso e copa definida, e palmeiras.

Os dados quantitativos foram anotados e processados em planilhas no Microsoft Excel® e foram calculados freqüência, abundância e índice Margalef.

Para o cálculo da freqüência, que exprime a regularidade da distribuição espacial de cada espécie nos parques, foi adotada a expressão (1):

$$
\operatorname{Freq}\left(\mathrm{sp}_{\mathrm{i}}\right)=\frac{\mathrm{NP}\left(\mathrm{sp}_{\mathrm{i}}\right)}{\mathrm{NPT}} 100(1)
$$

Onde:

Freq $\left(\mathrm{sp}_{\mathrm{i}}\right)$ : Freqüência da espécie i.

$\mathrm{NP}\left(\mathrm{sp}_{\mathrm{i}}\right)$ : Número de parques com a espécie i

NPT : Número total de parques. 
A abundância absoluta expressa o número de indivíduos das diferentes espécies na arborização dos parques e o Índice de riqueza de Margalef foi calculado pela expressão pela expressão (2):

$$
\mathrm{D}_{\mathrm{Mg}}=\frac{\mathrm{S}-1}{\ln \mathrm{N}}
$$

Onde:

$\mathrm{S}=$ número de espécies.

InN = logaritmo natural do número total de indivíduos

\section{RESULTADOS E DISCUSSÕES}

\section{Florística, abundância e freqüência de espécies de árvores e arvoretas}

Nos sete parques urbanos foram encontradas 87 morfoespécies de árvores e arvoretas, sendo 85 identificadas pelo menos ao nível de gênero, que se distribuíram em 35 famílias botânicas. As famílias de maior riqueza foram Caesalpiniaceae, com 11 espécies, Mimosaceae, com 10 espécies, seguidas de Myrtaceae (7 espécies), Bignoniaceae e Moraceae (essas últimas com 5 espécies cada).

Foram registrados 2087 exemplares de árvores e arvoretas nos sete parques. As espécies mais abundantes foram sombreiro (Clitoria fairchildiana), mangueira (Mangifera indica), pau d'arquinho (Tecoma stans), castanhola (Terminalia catappa) e ipê-roxo (Tabebuia impetiginosa) que reuniram $26,5 \%$ de todos os indivíduos.

Quarenta e uma espécies foram consideradas raras, com abundância entre 1 a 7 indivíduos, representando $47 \%$ das espécies encontradas. Por outro lado, apenas sete espécies podem ser consideradas como muito abundantes, com mais de 70 indivíduos, e juntas representam um terço de todas as árvores e arvoretas encontradas nos parques recifenses.

A distribuição das espécies mais abundantes não foi regular, como se observa na Tabela 1. A grande concentração de $C$. fairchildiana foi encontrada no Parque da Jaqueira enquanto Tecoma stans e Terminalia catappa deveram sua elevada abundância aos plantios realizados no Parque 13 de Maio. 
Tabela 1. Espécies de árvores e arvoretas encontradas nos parques urbanos de Recife, com respectivas abundâncias (N), freqüências (Freq) e número de tombamento no Herbário Sérgio Tavares (UFRPE)

1 - Parque 13 de Maio, 2 - Parque da Jaqueira, 3 - Parque Arraial Velho do Bom Jesus (Sitio da Trindade) , 4 - Parque de Santana, 5 - Parque Arraial Novo do Bom Jesus (Parque do Forte), 6 - Parque Arnaldo Assunção, 7 - Parque Robert Kennedy.

\begin{tabular}{|c|c|c|c|c|c|c|c|c|c|c|c|c|}
\hline \multirow[t]{2}{*}{ Espécie } & \multirow[t]{2}{*}{ Família } & \multirow{2}{*}{ Nome popular } & \multicolumn{7}{|c|}{ Número de indivíduos por parque } & \multirow{2}{*}{$\mathrm{N}$} & \multirow{2}{*}{$\begin{array}{c}\text { Freq } \\
\%\end{array}$} & \multirow{2}{*}{$\begin{array}{c}\text { № } \\
\text { HST }\end{array}$} \\
\hline & & & 1 & 2 & 3 & 4 & 5 & 6 & 7 & & & \\
\hline Clitoria fairchildiana R. A. Howard & Fabaceae & sombreiro & 20 & 86 & 5 & 23 & 4 & 1 & 11 & 150 & 100,0 & 12139 \\
\hline Mangifera indica L. & Anacardiaceae & mangueira & 60 & 27 & 30 & 8 & 6 & 2 & 7 & 140 & 100,0 & 12148 \\
\hline Tecoma stans (L.) Juss. ex Kunth & Bignoniaceae & pau d'arquinho & 90 & 2 & & & & 2 & & 94 & 42,9 & 12132 \\
\hline Terminalia catappa L. & Combretaceae & castanhola & 26 & 9 & 4 & 9 & 10 & 14 & 15 & 87 & 100,0 & 12147 \\
\hline Tabebuia impetiginosa (Mart. ex DC.) Standl. & Bignoniaceae & ipê-roxo & 8 & 19 & 3 & 15 & 5 & 30 & 2 & 82 & 100,0 & 12217 \\
\hline Delonix regia (Bojer ex Hook.) Raf. & Caesalpiniaceae & flamboyant & 19 & 53 & & & & & 1 & 73 & 42,9 & 12144 \\
\hline Syzygium cumini (L.) Skeels. & Myrtaceae & azeitona-roxa & 2 & 13 & 16 & 27 & 1 & 4 & 10 & 73 & 100,0 & 12161 \\
\hline Casuarina equisetifolia L. & Casuarinaceae & casuarina & & 55 & & 6 & 4 & & & 65 & 42,9 & 12172 \\
\hline Pachira aquatica Aubl. & Bombacaceae & carolina & 14 & 1 & 23 & 21 & 4 & & & 63 & 71,4 & 12140 \\
\hline Thevetia peruviana (Pers.) K. Schum. & Apocynaceae & chapéu-de-Napoleão & & 19 & 9 & & 13 & 21 & & 62 & 57,1 & 12167 \\
\hline Plumeria rubra L. & Apocynaceae & jasmim-vapor & 54 & 3 & & & & 2 & & 59 & 42,9 & 12131 \\
\hline Artocarpus integrifolia L. f. & Moraceae & jaca & 2 & 14 & 40 & 2 & 1 & & & 59 & 71,4 & 12165 \\
\hline Manilkara zapota (L.) P. Royen & Sapotaceae & sapoti & 23 & 23 & 10 & & & & 1 & 57 & 57,1 & 12146 \\
\hline Filicium decipiens (Wight \& Arn.) Thwaites & Sapindaceae & felício & 31 & 18 & & & & 7 & & 56 & 42,9 & 12197 \\
\hline Murraya exotica L. & Rutaceae & jasmim-laranjeira & 10 & 44 & & & & & & 54 & 28,6 & 12169 \\
\hline Caesalpinia echinata Lam. & Caesalpiniaceae & pau-brasil & 24 & 12 & & & 4 & 3 & 6 & 49 & 71,4 & 12159 \\
\hline Cassia javanica L. & Caesalpiniaceae & cássia-rosa & 13 & 6 & 2 & 13 & & 13 & 1 & 48 & 85,7 & 12196 \\
\hline $\begin{array}{l}\text { Tabebuia aurea (Silva Manso) Benth. \& Hook. } \\
\text { f. ex S. Moore }\end{array}$ & Bignoniaceae & craibeira & 13 & 10 & 1 & 4 & 9 & 8 & & 45 & 85,7 & 12321 \\
\hline Tabebuia sp & Bignoniaceae & ipê-rosa & & 44 & & & & & & 44 & 14,3 & 12428 \\
\hline Senna siamea (Lam.) H. S. Irwin \& Barneby & Caesalpiniaceae & cássia-siamea & 3 & 1 & 2 & 15 & 5 & & 17 & 43 & 85,7 & 12142 \\
\hline
\end{tabular}

continua..... 
Continuação Tabela 1

\begin{tabular}{|c|c|c|c|c|c|c|c|c|c|c|c|c|}
\hline \multirow[t]{2}{*}{ Espécie } & \multirow[t]{2}{*}{ Família } & \multirow[t]{2}{*}{ Nome popular } & \multicolumn{7}{|c|}{$\begin{array}{l}\text { Número de indivíduos por } \\
\text { parque }\end{array}$} & \multirow[t]{2}{*}{$\mathrm{N}$} & \multirow{2}{*}{$\begin{array}{c}\text { Freq } \\
\%\end{array}$} & \multirow{2}{*}{$\begin{array}{l}\text { № } \\
\text { HST }\end{array}$} \\
\hline & & & 1 & 2 & 3 & 4 & 5 & 6 & 7 & & & \\
\hline Spondias mombin L. & Anacardiaceae & cajá & 3 & 18 & 18 & 3 & & & & 42 & 57,1 & 12155 \\
\hline Lagerstroemia speciosa (L.) Pers. & Lythraceae & lagestroemia & 10 & 19 & & & 6 & 5 & & 40 & 57,1 & 12323 \\
\hline Tamarindus indica $\mathrm{L}$. & Caesalpiniaceae & tamarindo & 2 & 19 & 1 & 4 & 8 & 5 & & 39 & 85,7 & 12154 \\
\hline Caesalpinia peltophoroides Benth. & Caesalpiniaceae & sibipiruna & 3 & 13 & 1 & & 6 & 12 & & 35 & 71,4 & 12157 \\
\hline Cassia grandis L.f. & Caesalpiniaceae & cássia-rosa-gigante & 13 & 1 & 1 & 1 & & 19 & & 35 & 71,4 & 12198 \\
\hline Syzygium malaccense (L.) Merr. \& L. M. Perry & Myrtaceae & jambo-vermelho & 15 & 9 & 1 & 3 & 1 & & 2 & 31 & 85,7 & 12152 \\
\hline Cassia fistula L. & Caesalpiniaceae & cássia-cordão-de-ouro & 4 & 22 & 1 & & & & 3 & 30 & 57,1 & 12135 \\
\hline Ficus benjamina $L$. & Moraceae & fícus & 25 & 1 & & & & & & 26 & 28,6 & 12240 \\
\hline Schinus terebinthifolius Raddi & Anacardiaceae & aroeira & 3 & 4 & 17 & 2 & & & & 26 & 57,1 & 12156 \\
\hline Inga vera Willd. & Mimosaceae & ingá & & & 24 & 1 & & & & 25 & 28,6 & 12229 \\
\hline Spathodea campanulata P. Beauv. & Bignoniaceae & espatódia & 10 & 13 & & & & & 2 & 25 & 42,9 & 12143 \\
\hline Erythrina indica Lam. & Fabaceae & mulungu & 2 & 11 & & & 5 & 4 & 2 & 24 & 71,4 & 12174 \\
\hline Pterygota brasiliensis Allemão & Sterculiaceae & pau-rei & 18 & & & & 1 & & & 19 & 28,6 & 12223 \\
\hline Pithecellobium dulce (Roxb.) Benth & Mimosaceae & acácia-mimosa & 5 & 1 & 6 & 2 & 5 & & & 19 & 71,4 & 12149 \\
\hline Psidium guajava L. & Myrtaceae & goiaba & & 3 & & 3 & 2 & 1 & 8 & 17 & 71,4 & 12162 \\
\hline Eugenia uniflora L. & Myrtaceae & pitanga & & 7 & 8 & 1 & & & & 16 & 42,9 & 12178 \\
\hline Guarea macrophylla Vahl & Meliaceae & guarea & & & 15 & & & & & 15 & 14,3 & 12228 \\
\hline Anacardium occidentale L. & Anacardiaceae & caju & 3 & 9 & 1 & & & & 1 & 14 & 57,1 & 12150 \\
\hline Licania tomentosa & Chrysobalanacae & oiti & 4 & 3 & 4 & 2 & & & & 13 & 57,1 & 12215 \\
\hline Ziziphus joazeiro Mart. & Rhamnaceae & juazeiro & & & 12 & & & & & 12 & 14,3 & 12194 \\
\hline Albizia saman (Jacq.) F. Muell. & Mimosaceae & bordão-de-velho & 6 & & 6 & & & & & 12 & 28,6 & 12138 \\
\hline Sterculia foetida $\mathrm{L}$. & Sterculiaceae & xixá & 1 & 9 & & 2 & & & & 12 & 42,9 & 12160 \\
\hline Albizia lebbeck (L.) Benth. & Mimosaceae & acácia-branca & 7 & & 2 & & & & & 9 & 28,6 & 12129 \\
\hline Ficus elliotiana S. Moore & Moraceae & gameleira & & 4 & 5 & & & & & 9 & 28,6 & 12236 \\
\hline
\end{tabular}

continua.... 
Continuação Tabela 1

\begin{tabular}{|c|c|c|c|c|c|c|c|c|c|c|c|c|}
\hline \multirow{2}{*}{ Espécie } & \multirow{2}{*}{ Família } & \multirow{2}{*}{ Nome popular } & \multicolumn{7}{|c|}{ Número de indivíduos por parque } & \multirow{2}{*}{$\mathrm{N}$} & \multirow{2}{*}{$\begin{array}{c}\text { Freq } \\
\%\end{array}$} & \multirow{2}{*}{$\begin{array}{c}\text { № } \\
\text { HST }\end{array}$} \\
\hline & & & 1 & 2 & 3 & 4 & 5 & 6 & 7 & & & \\
\hline Couroupita guianensis Aubl. & Lecythidaceae & abricó-de-macado & 1 & 2 & & 6 & & & & 9 & 28,6 & 12130 \\
\hline Caesalpinia pulcherrima (L.) Sw. & Caesalpiniaceae & flamboianzinho & & 8 & & & & & & 8 & 14,3 & 12433 \\
\hline Bowdichia virgilioides Kunth & Fabaceae & sucupira & & & 5 & 2 & & & & 7 & 28,6 & 12225 \\
\hline Cecropia palmata Willd. & Cecropiaceae & imbaúba* & & & 6 & & & & & 6 & 14,3 & - \\
\hline Cedrela sp. & Meliaceae & cedro* & 6 & & & & & & & 6 & 14,3 & - \\
\hline Eucalyptus citriodora Hook. & Myrtaceae & eucalipto* & & 6 & & & & & & 6 & 14,3 & - \\
\hline Ficus elastica Roxb. & Moraceae & ficus & & 6 & & & & & & 6 & 14,3 & 12173 \\
\hline Averrhoa carambola L. & Oxalidaceae & carambola & & 6 & & & & & & 6 & 14,3 & 12158 \\
\hline Apeiba tibourbou Albl. & Tiliaceae & pau-de-jangada & & & & 5 & & & & 5 & 14,3 & 12164 \\
\hline Desconhecida 1 & - & - & & & 5 & & & & & 5 & 14,3 & - \\
\hline Triplaris americana $\mathrm{L}$. & Polygonaceae & gouguéia & 5 & & & & & & & 5 & 14,3 & 12239 \\
\hline Adansonia digitata L. & Bombacaceae & baobá & & 2 & & 1 & & 1 & & 4 & 42,9 & 12218 \\
\hline Bauhinia monandra Kurz & Caesalpiniaceae & pata-de-vaca & & & & & 4 & & & 4 & 14,3 & 12220 \\
\hline Malpighia glabra L. & Malpighiaceae & acerola & & 1 & & 3 & & & & 4 & 28,6 & 12170 \\
\hline Eugenia luschnathiana Klotzsch ex O. Berg & Myrtaceae & ubaia & 3 & 1 & & & & & & 4 & 28,6 & 12224 \\
\hline Coccoloba mollis Casar. & Polygonaceae & cocoloba & 1 & 3 & & & & & & 4 & 28,6 & 12234 \\
\hline Thespesia populnea (L.) Sol. ex Corrêa & Malvaceae & algodão-do-Pará & 1 & & & & 2 & & 1 & 4 & 42,9 & 12226 \\
\hline Annona squamosa L. & Annonaceae & pinha & & 3 & & & & & & 3 & 14,3 & 12188 \\
\hline Hymenaea sp. & Caesalpiniaceae & jatobá* & & 3 & & & & & & 3 & 14,3 & \\
\hline Machaerium angustifolium Vogel & Fabaceae & espinheiro & & & 3 & & & & & 3 & 14,3 & 12482 \\
\hline Azadirachta indica A. Juss. & Meliaceae & nim & & 3 & & & & & & 3 & 14,3 & 12184 \\
\hline Leucaena leucocephala (Lam.) de Wit & Mimosaceae & leucena & 2 & & 1 & & & & & 3 & 28,6 & 12153 \\
\hline Calliandra brevipes Benth. & Mimosaceae & escova-de-peru & 3 & & & & & & & 3 & 14,3 & 12136 \\
\hline Artocarpus altilis (Parkinson) Fosberg & Moracae & fruta-pão & & 2 & 1 & & & & & 3 & 28,6 & 12432 \\
\hline Desconhecida 2 & - & - & & 3 & & & & & & 3 & 14,3 & - \\
\hline
\end{tabular}


Continuação Tabela 1

\begin{tabular}{|c|c|c|c|c|c|c|c|c|c|c|c|c|}
\hline \multirow{2}{*}{ Espécie } & \multirow{2}{*}{ Família } & \multirow{2}{*}{ Nome popular } & \multicolumn{7}{|c|}{ Número de indivíduos por parque } & \multirow{2}{*}{$\mathrm{N}$} & \multirow{2}{*}{$\begin{array}{c}\text { Freq } \\
\%\end{array}$} & \multirow{2}{*}{$\begin{array}{c}\text { № } \\
\text { HST }\end{array}$} \\
\hline & & & 1 & 2 & 3 & 4 & 5 & 6 & 7 & & & \\
\hline Crataeva tapia L. & Capparaceae & trapiá & & & & 2 & & & & 2 & 14,3 & 12734 \\
\hline Persea americana Mill. & Lauraceae & abacateiro & & 2 & & & & & & 2 & 14,3 & 12325 \\
\hline Albizia inundata (Mart.) Barneby \& J.W. Grimes & Mimosaceae & * & 1 & & 1 & & & & & 2 & 28,6 & - \\
\hline Genipa americana L. & Rubiaceae & jenipapo & & & 1 & 1 & & & & 2 & 14,3 & 12195 \\
\hline Syzygium aqueum (Burm. f.) Alston & Myrtaceae & jambo-branco & & & 2 & & & & & 2 & 14,3 & 12227 \\
\hline Talisia esculenta (A. St.-Hil.) Radlk. & Sapindaceae & pitomba & & 1 & & 1 & & & & 2 & 28,6 & 12163 \\
\hline Simarouba amara Aubl. & Simaroubaceae & praiba & & & & 2 & & & & 2 & 14,3 & 12213 \\
\hline Annona montana Macfad. & Annonaceae & araticum & & & & & 1 & & & 1 & 14,3 & 12219 \\
\hline Araucaria excelsa (Lamb.) R. Br & Araucariaceae & pinheiro * & & 1 & & & & & & 1 & 14,3 & - \\
\hline Ceiba pentandra (L.) Gaertn. & Bombacaceae & $\begin{array}{l}\text { barriguda-de- } \\
\text { espinho }\end{array}$ & & 1 & & & & & & 1 & 14,3 & 12179 \\
\hline Muntingia calabura L. & Elaeocarpaceae & calabura & & 1 & & & & & & 1 & 14,3 & 12177 \\
\hline Adenanthera pavonina L. & Mimosaceae & olho-de-pombo & 1 & & & & & & & 1 & 14,3 & 12141 \\
\hline Anadenanthera colubrina (Vell.) Brenan & Mimosaceae & angico* & & & 1 & & & & & 1 & 14,3 & - \\
\hline Morus nigra L. & Moraceae & amora-preta & & 1 & & & & & & 1 & 14,3 & 12427 \\
\hline Citrus sinensis (L.) Osbeck & Rutaceae & laranjeira* & & 1 & & & & & & 1 & 14,3 & - \\
\hline Chrysophyllum cainito L. & Sapotaceae & cainito & 1 & & & & & & & 1 & 14,3 & 12145 \\
\hline Theobroma cacao L. & Sterculiaceae & cacau & & 1 & & & & & & 1 & 14,3 & 12175 \\
\hline
\end{tabular}

${ }^{*}$ Material estéreo identificado, mas não foi introduzido no HST 
Tabela 2 . Espécies de palmeiras encontradas nos sete parques urbanos de Recife, com respectivas abundâncias ( $N$ ), freqüências (Freq) e número de tombamento no Herbário Sérgio Tavares (UFRPE)

1 - Parque 13 de Maio, 2 - Parque da Jaqueira, 3 - Parque Arraial Velho do Bom Jesus (Sitio da Trindade) , 4 - Parque de Santana, 5 - Parque Arraial Novo do Bom Jesus (Parque do Forte), 6 - Parque Arnaldo Assunção, 7 - Parque Robert Kennedy.

\begin{tabular}{|c|c|c|c|c|c|c|c|c|c|c|c|}
\hline \multirow[t]{2}{*}{ Espécie } & \multirow[t]{2}{*}{ Nome popular } & \multicolumn{7}{|c|}{ Número de indivíduos no parque } & \multirow[b]{2}{*}{$\mathrm{N}$} & \multirow{2}{*}{$\begin{array}{c}\text { Freq. } \\
\%\end{array}$} & \multirow{2}{*}{ № HST } \\
\hline & & 1 & 2 & 3 & 4 & 5 & 6 & 7 & & & \\
\hline Dypsis lutescens (H. Wendl.) Beentje \& J. Dransf. & palmeira-de-sala & 163 & 7 & & & & 5 & & 175 & 42,86 & 12242 \\
\hline Roystonea oleracea (Jacq.) O. F. Cook & palmeira-imperial & 75 & 30 & 3 & 10 & 3 & 10 & 32 & 163 & 100,00 & 12244 \\
\hline Cocos nucifera L. & coqueiro & 56 & 7 & 24 & 19 & 7 & 1 & & 114 & 85,71 & 12248 \\
\hline Acrocomia intumescens Drude & macaúba & 12 & 34 & 4 & 4 & 6 & 27 & & 87 & 85,71 & 12243 \\
\hline Elaeis guineensis Jacq. & dendê & 40 & & 7 & 3 & 2 & 29 & & 81 & 71,43 & 12247 \\
\hline $\begin{array}{l}\text { Ptychosperma macarthurii (H. Wendl. ex H. J. Veitch) H. Wendl. } \\
\text { ex Hook. f. }\end{array}$ & palmeira-sabu & 41 & & & & & 1 & & 42 & 28,57 & 12246 \\
\hline Pritchardia pacifica Seem. \& H. Wendl. & palmeira-leque & 16 & 3 & & & & & & 19 & 28,57 & 12249 \\
\hline Caryota mitis Lour. & palmeira-mulambo & 3 & 1 & & & & 13 & & 17 & 42,86 & 12245 \\
\hline Livistona rotundifolia (Lam.) Mart. & palmeira-japonesa & & 8 & & & & & & 8 & 14,29 & 12334 \\
\hline Desconhecida 1 & - & & & 7 & & & & & 7 & 14,29 & - \\
\hline Phoenix roebelenii O'Brien & palmeira-fênix & 1 & 3 & & & & & & 4 & 28,57 & 12333 \\
\hline Sabal sp & palmeira sabal & 2 & & & & & & & 2 & 28,57 & - \\
\hline Phoenix reclinata Jacq. & palmeira-tâmara & 1 & & & & & & & 1 & 14,29 & 12250 \\
\hline Borassus aethiopium Mart. & palmeira-africana* & & & 1 & & & & & 1 & 14,29 & - \\
\hline
\end{tabular}

${ }^{*}$ Material estéreo identificado, mas não foi introduzido no HST 
A alta densidade de sombreiros em um parque é desaconselhável, dada a susceptibilidade de grandes populações homogêneas à ocorrência de pragas e doenças. (SILVA, 2005). A incidência periódica de um psilídeo (Euphalerus clitoriae) que causa desfolhamento das árvores, com prejuízos ao seu estado geral, é uma expressão desse problema no parque. Por outro lado, plantios mais recentes foram observados, predominantemente de Tabebuia sp, de forma a reduzir, no futuro, a população de Clitoria fairchildiana.

As espécies mais freqüentes nos parques urbanos de Recife estiveram também entre as mais abundantes (C. fairchildiana, M. indica, T.catappa, T. impetiginosa $e$ Syzygium cumini - azeitona-roxa ou jambolão), existentes em todos os sete parques. Fruteiras tradicionais cultivadas (Mangifera indica, Syzygium cumini, Artocarpus integrifolia e Syzygium malaccense) foram bastante empregadas na arborização dos parques, apesar dos inconvenientes causados pela queda e furto de frutos.

O Arraial Velho do Bom Jesus teve a jaqueira (Artocarpus integrifolia), fruteira exótica, como a espécie mais abundante, retratando a vegetação dos antigos sítios recifenses. Diferentemente dos outros parques, o Arraial Velho apresentou espécies nativas pouco usuais no paisagismo entre as mais abundantes: Inga vera (ingá), Spondias mombin (cajá) e Schinus terebinthifolius (aroeira-da-praia). Esse parque destacou-se pela presença, ainda que em pequena quantidade, de outras arbóreas nativas raras na arborização urbana como sucupira (Bowdichia virgilioides) e cocoloba (Coccoloba mollis), merecendo especial atenção o cuidado com a conservação e reprodução dessas árvores. Foi o único parque onde se encontraram Zizyphus joazero, Guarea macrophylla, Cecropia palmata, Machaerium angustifolium e Anadenanthera colubrina.

No Parque de Santana, às margens do rio Capibaribe recobertas por manguezal que se integra à paisagem do parque, a espécie mais abundante encontrada foi a azeitona-roxa (Syzygium cuminii) de procedência asiática. Sombreiro (C. fairchildiana) mais uma vez destacou-se com elevada abundância, assim como a carolina ( $P$. aquatica). Cássia siamea (ou cássia amarela, Senna siamea), exótica muito empregada na arborização de calçadas em Recife, também esteve entre as mais abundantes.

Apesar da pequena quantidade de espécies, este parque se destacou por apresentar espécies raras na arborização como ingá-branco (Inga laurina), pau-de-jangada (Apeiba tibourbou), sucupira (Bowdichia virgilioides), trapiá (Crataeva tapia) e praiba (Simarouba amara), merecendo uma atenção especial à manutenção e reprodução dessas árvores pelo seu papel na informação e educação ambiental, pois são espécies que ocorrem naturalmente na zona do litoral e mata do estado de Pernambuco.

O Parque Arraial Novo do Bom Jesus apresentou cobertura arbórea escassa e algumas espécies exóticas foram mais abundantes como Terminalia catappa (castanhola) e 
Thevetia peruviana (chapéu-de-napoleão). Deve-se ter cautela quanto à utilização do chapéu-de-napoleão na arborização, pois essa espécie pertencente à família Apocynaceae apresenta toxicidade (SINITOX, 2005), sendo especialmente contra-indicada em parques, onde transitam crianças. Apesar disso, a espécie também foi bastante empregada no Parque da Jaqueira.

No parque Arnaldo Assunção foi encontrada a maior concentração de Tabebuia impetiginosa (ipê-roxo) dos parques recifenses, representando $19,5 \%$ das árvores e arvoretas lá existentes, ilustrando a opção por plantios quase que exclusivos de determinadas espécies.

\section{Florística, abundância e freqüência de palmeiras}

Foi registrada a presença de 721 indivíduos da família Arecaceae, destacando-se Dypsis lutescens (palmeira-de-sala), Roystonea oleracea (palmeira imperial), Cocos nucifera (coqueiro-da-Bahia), Acrocomia intumescens (macaíba) e Elaeis guineensis (dendezeiro). A palmeira imperial foi encontrada em todos os parques da cidade, com a abundância mais elevada no Parque 13 de Maio, que reúne $56,8 \%$ de todas as palmeiras encontradas (Tabela 2).

Apenas 14 espécies de palmeiras foram encontradas compondo a arborização dos parques urbanos, sendo que $86 \%$ dos indivíduos pertenciam às cinco espécies citadas. Apesar da enorme riqueza de espécies de palmeiras da flora brasileira, que the valeu o nome indígena de Pindorama (terra das palmeiras), apenas duas das espécies de palmeiras adotadas nos parques são nativas (Cocos nucifera e Acrocomia intumescens, segundo as procedências indicadas por LORENZI et al., 1996).

\section{Riqueza e densidade de árvores, arvoretas e palmeiras dos parques urbanos de Recife}

A maior abundância de árvores, arvoretas e palmeiras foi verificada no Parque Treze de Maio, com 981 indivíduos de 58 diferentes espécies, com índice de riqueza de Margalef $\left(D_{\mathrm{Mg}}\right)$ de 8,27 (Tabela 3). O Parque da Jaqueira, por sua vez, apresentou 773 indivíduos, pertencentes a 68 espécies, sendo o parque com maior riqueza expressa por elevado número de espécie e índice de riqueza de Margalef (10,07). Em termos de densidade por área, foram encontrados nesses dois parques, respectivamente, 142,2 e 110,4 exemplares de árvores, arvoretas e palmeiras por hectare. 
Tabela 3. Parques urbanos de Recife com suas respectivas áreas, números de árvores, arvoretas e palmeiras, riqueza de espécies e índice de riqueza de Margalef $\left(D_{M g}\right)$

\begin{tabular}{|c|c|c|c|c|c|c|c|c|c|}
\hline \multirow[b]{2}{*}{ PARQUES } & \multirow[b]{2}{*}{$\begin{array}{l}\text { ÁREA } \\
\text { (ha) }\end{array}$} & \multicolumn{4}{|c|}{ Número de indivíduos } & \multicolumn{3}{|c|}{ Número de espécies } & \multirow[b]{2}{*}{$\mathrm{D}_{\mathrm{Mg}}$} \\
\hline & & $\begin{array}{l}\text { Árvores e } \\
\text { arvoretas }\end{array}$ & Palmeiras & Total & N/ha & $\begin{array}{l}\text { Árvores e } \\
\text { arvoretas }\end{array}$ & Palmeiras & Total & \\
\hline $\begin{array}{l}1 \text { - Treze de } \\
\text { Maio }\end{array}$ & 6,9 & 571 & 410 & 981 & 142,2 & 46 & 11 & 58 & 8,27 \\
\hline 2 - Jaqueira & 7,0 & 680 & 93 & 773 & 110,4 & 60 & 8 & 68 & 10,07 \\
\hline $\begin{array}{l}3 \text { - Arraial } \\
\text { Velho do } \\
\text { Bom Jesus }\end{array}$ & 4,6 & 294 & 46 & 340 & 73,9 & 39 & 6 & 45 & 7,55 \\
\hline 4-Santana & 4,0 & 191 & 36 & 227 & 56,7 & 32 & 4 & 36 & 6,64 \\
\hline $\begin{array}{l}5 \text { - Arraial } \\
\text { Novo do } \\
\text { Bom Jesus }\end{array}$ & 3,0 & 107 & 18 & 125 & 41,7 & 23 & 4 & 27 & 5,38 \\
\hline $\begin{array}{l}6 \text { - Arnaldo } \\
\text { Assunção }\end{array}$ & 2,4 & 154 & 86 & 240 & 100,0 & 19 & 7 & 26 & 4,56 \\
\hline $\begin{array}{l}7 \text { - Robert } \\
\text { Kennedy }\end{array}$ & 1,8 & 90 & 32 & 122 & 67,8 & 17 & 1 & 18 & 3,54 \\
\hline Total & 29,7 & 2097 & 721 & 2808 & 94,5 & 87 & 14 & 101 & 12,59 \\
\hline
\end{tabular}

O parque menos arborizado foi o Robert Kennedy, com 122 exemplares de espécies de árvores, arvoretas e palmeiras (67,8 exemplares/ha). Por outro lado, relacionando-se os números de espécimes com a área dos parques, as piores condições de densidade foram encontradas nos parques Santana e Arraial Novo do Bom Jesus (56,7 e 41,7 indivíduos/ha, respectivamente).

O Parque Robert Kennedy, além de contar com um número menor de exemplares, apresentou índice de riqueza muito baixo $\left(D_{\mathrm{Mg}}=3,54\right)$, refletindo as elevadas proporções de Roystonea oleracea, Terminalia catappa e Senna siamea, que representaram 26,2; 13,9 e $12,3 \%$ dos exemplares, respectivamente, sendo as três espécies exóticas. Assim como no parque Arnaldo Assunção $\left(D_{M g}=4,56\right)$, o pequeno número de espécies empregadas e a excessiva dominância de poucas espécies refletiram-se no baixo índice de riqueza de espécies arbóreas, arbustivas e de palmeiras, fazendo com que não cumpram seu papel na conservação da diversidade vegetal e também pouco se aproveite das potencialidades paisagísticas das espécies.

As densidades de árvores, arvoretas e palmeiras foram consideradas baixas em quatro dos sete parques (Arraial Novo do Bom Jesus, Santana, Robert Kennedy e Arraial Velho do Bom Jesus), pois implicam em espaçamentos entre indivíduos superiores a $10 \mathrm{x}$ 10m. A densidade de árvores e seu porte estão relacionados ao conforto térmico e, portanto, ao uso dado ao parque pelos cidadãos e à sua função no ambiente urbano, tendo 
Gomes \& Amorim (2003) ressaltado a importância de espécies de grande porte na atenuação da radiação e na oferta de conforto térmico.

\section{CONCLUSÕES}

Recife tem poucos parques urbanos, concentrados em apenas sete dos seus 94 bairros, que representam uma fração muito pequena do território da cidade. O parque como maior densidade de árvores, arvoretas e palmeiras foi o Treze de Maio enquanto maior riqueza florística foi encontrada no Parque da Jaqueira. Nos demais parques identificaramse condições insatisfatórias de densidade ou de riqueza.

O potencial paisagístico da flora local, típica da Mata Atlântica e de seus ecossistemas associados, foi mal aproveitado nesses parques, privilegiando-se o plantio de espécies oriundas de outros biomas, como a amazônica Clitoria fairchildiana, e as espécies exóticas como mangueira (Mangifera indica), pau d'arquinho (Tecoma stans) e castanhola (Terminalia catappa), espécies mais abundantes nos parques da cidade.

Os parques Arraial Novo do Bom Jesus, Santana, Robert Kennedy e Arraial Velho do Bom Jesus precisam receber novos plantios de espécies arbóreas, respeitando-se evidentemente os princípios da boa composição paisagística, contribuindo para amenização climática, melhor qualidade de vida, conservação e valorização de flora nativa.

Além disso, novos parques arborizados precisam ser criados na cidade, integrando um sistema de áreas verdes que contemple várias categorias de parques, praças e jardins, e, especialmente nesses parques, deve-se priorizar o uso das espécies nativas dos ecossistemas associados ao domínio Mata Atlântica, onde Recife se situa.

\section{REFERÊNCIAS BIBLIOGRÁFICAS}

BRANDÃO, A. M. P. M.; LUCENA, A. J. A. A ilha térmica e sua influência no conforto humano na área central da cidade do Rio de Janeiro. In: Simpósio Brasileiro de Geografia Física Aplicada. Resumos. Belo Horizonte: UFMG p.66-67, 1999.

CASTRO, N. S. Importância da arborização no desempenho técnico da gerência de Coordenação Regional de Porto Alegre. Porto Alegre, 2000. 96p (Monografia de Especialização da Universidade Federal do Rio Grande do Sul).

GATTO, A. Solo, planta e água na formação de paisagem. Viçosa: Editora Aprenda Fácil. 144 p. 2002. 
GOMES, M. A. S.; AMORIM, M. C. C. T. Arborização e conforto térmico no espaço urbano: estudo de caso nas praças públicas de Presidente Prudente (SP). Caminhos de Geografia (Revista on-line). v.7, n. 10, p. 94-106., 2003. Disponível em <www.ig.ufu.br/caminhos_degeografia.html> Acesso em 21 de junho de 2007.

GUIA DO RECIFE. Disponível em <http://www.recifeguide.com/brasil/informacoes/climarecife-olinda.html>. Acesso em 14 de outubro de 2007.

LIRA, R. S. Arborização do Parque da Criança. In. Simpósio Brasileiro de Etnobiologia e Etnoecologia, 4, 2002. Resumos. Recife:SBEE, p.53 - 54, 2002.

LORENZI, L.; SOUZA, H. M. ; TORRES, M.A. ; BACHER, L. B. Árvores exóticas no Brasil: madeireiras, ornamentais e aromáticas. Nova Odessa, Instituto Plantarum. 2003.

LORENZI, H.; SOUZA, H. M.; MEDEIROS-COSTA, J. T..; BEHR, N. Palmeiras no Brasil: exóticas e nativas. Nova Odessa: Instituto Plantarum, 1996.

RECIFE: INFORMAÇÕES GERAIS. Recife: Prefeitura da Cidade de Recife - Secretaria de Planejamento, Urbanismo e Meio Ambiente, Departamento de Informações e Projeções. 2006. (Folheto informativo)

SÁ CARNEIRO, A. R.; MESQUITA, L. B.. Espaços livres do Recife. Recife: Prefeitura da Cidade do Recife / Universidade Federal de Pernambuco, 2000.

SILVA. F. R., Occurrence and biological aspects of the Clitoria tree psyllid in Brazil. Scientiae Agricola, Piracicaba, v.62, n.3, p.281-285, 2005.

SINITOX Site do Sistema Nacional de Informações Tóxico-Farmacológicas. Disponível em: <http://www.fiocruz.br/sinitox/prognacional.htm> Acesso em 9 de novembro de 2005. 